\title{
Claudin 10 acts as a novel biomarker for the prognosis of patients with ovarian cancer
}

\author{
ZHONGJUN LI ${ }^{1,2}$, WENTING XUAN ${ }^{1}$, LISHAN HUANG ${ }^{1}$, NIANKUN CHEN ${ }^{1,2}$, \\ ZHIYONG HOU ${ }^{1}$, BIYAN LU ${ }^{3}$, CHUANGYU WEN $^{1}$ and SURAN HUANG ${ }^{1}$ \\ ${ }^{1}$ Department of Obstetrics and Gynecology, Dongguan Affiliated Hospital, Southern Medical University, Dongguan, \\ Guangdong 523059; ${ }^{2}$ Department of Obstetrics and Gynecology, Nanfang Hospital, Southern Medical University, \\ Guangzhou, Guangdong 510515; ${ }^{3}$ Department of Basic Medical Sciences, Dongguan Polytechnic, \\ Dongguan, Guangdong 523808, P.R. China
}

Received September 10, 2019; Accepted March 9, 2020

DOI: $10.3892 / \mathrm{ol} .2020 .11557$

\begin{abstract}
Ovarian cancer (OC) is one of the most fatal gynecological malignancies in the world and confers a poor 5-year survival rate. The present study was designed to discover novel prognostic markers for patients with $\mathrm{OC}$ in order to estimate disease metastasis or recurrence. Based on the large cohorts of transcriptome data from multicenter sources, a comprehensive analysis was performed to explore potential prognostic markers. A total of 269 differentially expressed genes were identified, of which 32 were upregulated and 237 downregulated in OC tissues compared with the corresponding expression in normal tissues. Kaplan-Meier analysis, log-rank test and nomogram analysis were employed to demonstrate that low expression levels of claudin 10 (CLDN10) were associated with a less favorable disease prognosis. The most promising prognostic marker for OC was subsequently selected. Additionally, the prognostic nomogram was constructed in order to assess the 5-year survival rate using CLDN10 expression as a prognostic marker for OC. Furthermore, gene set enrichment analysis and analysis of the tumor-associated competing endogenous RNA network were performed to elucidate the potential biological processes associated with CLDN10 expression. The current results indicated that CLDN10 may influence OC progression via transforming growth factor- $\beta$ (TGF- $\beta$ )- or
\end{abstract}

Correspondence to: Professor Suran Huang, Department of Obstetrics and Gynecology, Dongguan Affiliated Hospital, Southern Medical University, 3 Wangdao Road, Dongguan, Guangdong 523059, P.R. China

E-mail: suranhuangdg@163.com

Abbreviations: OC, ovarian cancer; CLDN10, claudin 10; GSEA, gene set enrichment analysis; ceRNA, competing endogenous RNA; GEO, Gene Expression Omnibus; DEGs, differentially expressed genes; KEGG, Kyoto Encyclopedia of Genes and Genomes

Key words: claudin 10, ovarian cancer, prognosis, signaling pathways, competing endogenous RNA
WNT/ $\beta$-catenin-induced epithelial-to-mesenchymal transition (EMT). The associations among CLDN10, microRNA-486-5p, TGF- $\beta$, WNT/ $\beta$-catenin and EMT should be further investigated in future studies.

\section{Introduction}

Ovarian cancer (OC) is one of the most frequent cancer types among female patients. There were a total of 295,414 new OC cases in 2018 that resulted in 184,799 deaths worldwide (1). OC is a highly heterogeneous disease, and epithelial OC has been divided into four histological subtypes, including endometrioid, mucinous, serous and clear cell adenocarcinoma (2). OC is considered a mixture of these representative histological types with different molecular etiologies, tumor progression features and disease prognoses. This hinders the efficacy of current treatments for OC, such as platinum-based combination chemotherapy, surgery and neoadjuvant chemotherapy $(3,4)$. Unfortunately, the majority of patients with OC $(>70 \%)$ are diagnosed at an advanced or metastatic stage (III or IV) and their 5-year overall survival rate is $\sim 40 \%$, while the 5-year overall survival rate of patients with stage I OC is $\sim 90 \%(5,6)$. At present, no effective screening approaches have been reported that can be used effectively to decrease the mortality rate of OC, due to low sensitivity and specificity (7). Prognostic biomarkers with high sensitivity and specificity can be used to estimate disease metastasis or recurrence, classify patients at different risk levels for corresponding outcome assessment and guide clinical treatments, in this way reducing the mortality rate of patients with OC (8). Therefore, it is crucial to identify novel prognostic biomarkers to monitor cancer progression, overall therapeutic efficiency and risk stratification in patients with OC.

During the past decades, high-throughput sequencing technology has been used for the investigation of several types of cancer. This method can aid the identification of specific patterns for cancer diagnosis, classification and therapeutic response $(9,10)$. A large amount of core data obtained by high-throughput sequencing technology has been stored in public databases. These data can be integrated and re-analyzed in order to uncover important targets that can be used for cancer 
therapy. In the present study, the GSE66957, GSE6008 and GSE26712 datasets were selected from the Gene Expression Omnibus (GEO) database to identify differentially expressed genes (DEGs). In the present study, a total of 269 DEGs were discovered by bioinformatic analysis (differentially expressed in at least 2 datasets), and the most promising prognostic marker for OC was selected for further study. The expression levels of this gene in OC tissues compared with those in normal tissues were analyzed using the GEO datasets and confirmed using quantitative PCR. A predictive nomogram with high accuracy was established for prognosis prediction. Gene set enrichment analysis (GSEA) and analysis of the tumor-associated competing endogenous RNA (ceRNA) network were used to clarify the potential biological processes associated with this gene.

\section{Materials and methods}

Patients and tissue samples. A total of $82 \mathrm{OC}$ tissues (from OC patients who underwent debulking surgery) and 82 normal ovarian tissues (from hysteromyoma patients who underwent hysterectomy and oophorectomy) were obtained from the Department of Obstetrics and Gynecology at Dongguan Affiliated Hospital, Southern Medical University between July 2014 and December 2017. OC tissues included 47 serous adenocarcinomas, 9 mucinous adenocarcinomas, 6 endometrioid adenocarcinomas, 6 clear-cell tumors, 6 germ-cell tumors and 8 other types. The mean age of the patients was 52.96 years, ranging from 17 to 86 years. None of the recruited patients in the present study had received preoperative radiotherapy or preoperative chemotherapy. Written informed consent was obtained from patients. This study was approved by the Regional Institutional Review Board of Dongguan Affiliated Hospital, Southern Medical University and carried out according to the Declaration of Helsinki. All fresh tumor tissue specimens were cryopreserved immediately in liquid nitrogen and stored at $-80^{\circ} \mathrm{C}$ until further use.

GEO datasets and the cancer genome atlas (TCGA) database. GEO (https://www.ncbi.nlm.nih.gov/geo/query/acc.cgi) is a public database containing experiments and gene expression profiles that can be downloaded and used for subsequent analysis. The expression profiles of the transcriptomes from the following datasets were systematically analyzed to ensure the credibility of the present study: GSE66957 (n=69; Moffitt Cancer Center, Biostatistics and Bioinformatics), GSE6008 ( $n=103$; University of Michigan) and GSE26712 ( $n=195$; University of Michigan) (11-14). The sequencing data were collected on Affymetrix (Thermo Fisher Scientific, Inc.) microarray and Illumina, Inc., platforms by different researchers. The clinicopathological characteristics of 266 patients with OC were collected from TCGA database via the cBioPortal (http://www.cbioportal.org), including the 7th American Joint Committee on Cancer (AJCC) staging indices (https://www. nationwidechildrens.org/research/resources-infrastructure/core facilities/biospecimen-core-resource/the-cancer-genome-atlas/tcgaforms-and-documents), the disease-free survival time, the overall survival time, the quality of life (https:/www.nationwidechildrens.org/research/resources-infrastructure/core facilities/ biospecimen-core-resource/the-cancer-genome-atlas/tcga-formsand-documents) and the recurrence time. The clinical stages of
OC were divided into 4 stages (I, II, III and IV) according to the origin, pathogenesis and prognosis by FIGO criteria $(15,16)$.

Identification of DEGs. DEGs were analyzed via the $\mathrm{R}$ (v3.34) limma package (http://www.bioconductor.org/packages/release/bioc/html/limma.html) between OC and normal tissues. The different levels of gene expression between $\mathrm{OC}$ and normal tissues were evaluated by the $\log 2$ fold-change. Genes with a $\log 2$ fold-change $>1$ (absolute value) and $\mathrm{P}<0.05$ were selected as candidate signature genes for further analysis. A Venn plot was constructed for identification of reliable OC-associated genes.

Kaplan-Meier plot. The Kaplan-Meier method was used to evaluate the association between the survival time of patients with OC and the DEGs selected from the Venn plot collection. The genes with the most significantly different log-rank P-values were selected as the candidate genes. The difference in the expression of the candidate genes was analyzed via the GEO datasets, as well as the prognostic value of the candidate genes.

Quantitative PCR ( $q P C R)$ assay. Total RNA was extracted from the tumor tissue specimens of the patients with $\mathrm{OC}$ using the RNA isolation kit (BioTeke Corporation) according to the manufacturer's protocol. CLDN10 mRNA expression levels were assessed by qPCR using a SYBR Green PCR kit (Takara Biotechnology Co., Ltd.). The reaction conditions were as follows: Preincubation at $95^{\circ} \mathrm{C}$ for $10 \mathrm{~min}$, followed by 40 cycles of $95^{\circ} \mathrm{C}$ for $10 \mathrm{sec}, 60^{\circ} \mathrm{C}$ for $10 \mathrm{sec}$, and $72^{\circ} \mathrm{C}$ for $20 \mathrm{sec}$. CLDN10 expression levels were normalized to those of GAPDH mRNA levels ( $\triangle \mathrm{Cq}=\mathrm{Cq}$ CLDN10-Cq GAPDH) and was calculated using $2^{-\Delta \Delta C q}$ between two groups (17). The primers for CLDN10 and GAPDH detection were as follows: CLDN10 forward, 5'-CTGTGGAAGGCGTGCGTTA-3' and reverse, 5'-CAAAGAAGCCCAGGCTGACA-3'; and GAPDH forward, 5'-GTCTCCTCTGACTTCAACAGCG-3' and reverse, 5'-ACCACCCTGTTGCTGTAGCCAA-3'.

Prognostic nomogram construction. A nomogram was established to predict the 5-year survival rate. The prognostic significance of the parameters was evaluated by multivariate Cox proportional hazard regression analysis. CLDN10 expression was used as a novel variable for the establishment of the nomogram. For other variables, several survival-associated indices and basic medical information were selected, including age, AJCC staging indices and lymphovascular invasion index. The predictive accuracy of the nomogram for the 5-year survival rate was assessed via the concordance index (C-index), which was estimated by the area under the receiver operating characteristic (ROC) curve for predictive probability. Additionally, the ROC curve of conventional features for predicting the 5-year survival rate was displayed for comparison. The calibration was conducted by comparing the nomogram predicted probabilities with the observed probabilities. The univariate and multivariate Cox proportional hazards tests were performed for conventional clinical feature analysis.

ceRNA network construction. A CLDN10-associated ceRNA network was constructed to reveal the potential biological 
A

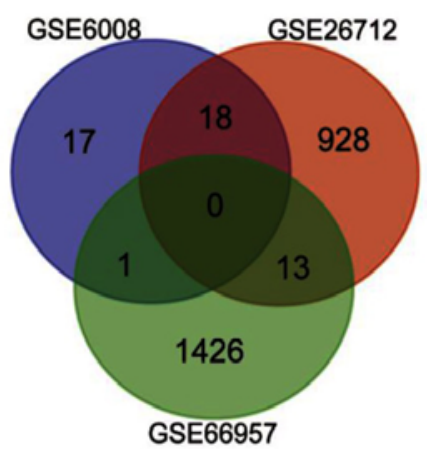

D

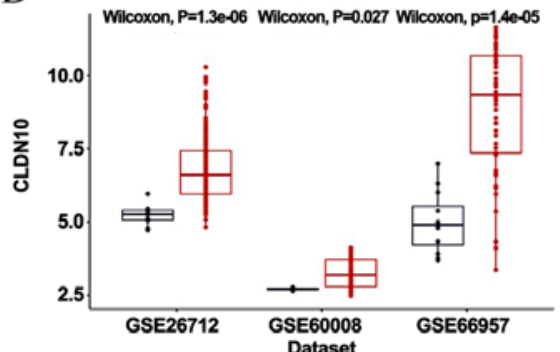

B

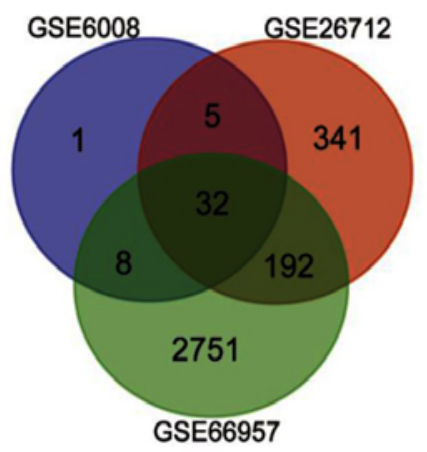

E

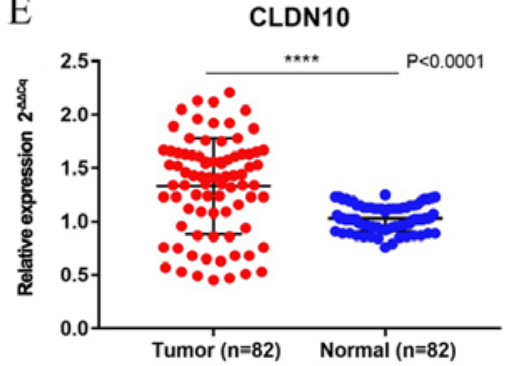

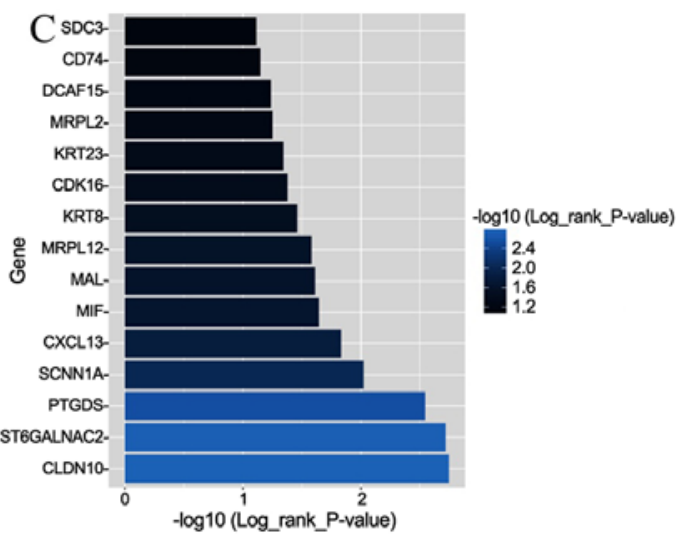

F $\quad$ CLDN10

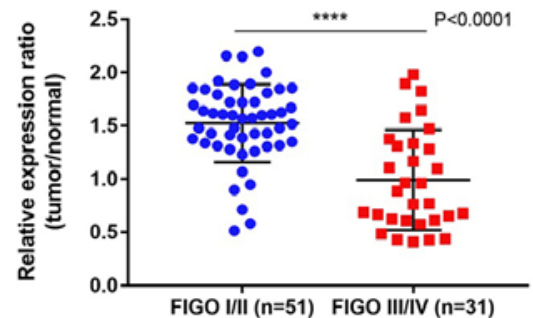

Figure 1. Identification of DEGs and candidate genes. (A) Venn plot of upregulated DEGs via log2 fold-change. (B) Venn plot of downregulated DEGs via $\log 2$ fold-change. (C) Log-rank P-value of 15 selected DEGs ( $\mathrm{P}<0.05$ ). (D) CLDN10 expression differences between tumor and normal tissues by analyzing multicenter data sources. CLDN10 was upregulated in OC tissues. (E) Quantitative PCR analysis of CLDN10 expression in 82 ovarian tumor and 82 normal ovarian tissues. (F) CLDN10 was upregulated in FIGO I/II tumor tissues compared with that in FIGO III/IV tumor tissues. ${ }^{* * * * *}$ P $<0.0001$. DEGs, differentially expressed genes; CLDN10, claudin 10; FIGO, International Federation of Gynecology and Obstetrics.

processes and associations between CLDN10 and other RNAs, including long non-coding RNAs (IncRNAs), circular RNAs (circRNA) and miRNAs. miRTarBase (http://mirtarbase.mbc. nctu.edu.tw/php/index.php) was used to elucidate the association between CLDN10 and miRNA expression. This database includes a record of experimental validated miRNA-mRNA associations. miRNAs with at least one experimental validation were selected. In addition, the associations among selected miRNAs, circRNAs and IncRNAs were revealed via Starbase v.2.0 (http://starbase.sysu.edu.cn/starbase2/index. php). The ceRNA network was created using Cytoscape (v.3.5.1) (https://cytoscape.org).

GSEA. GSEA and Kyoto Encyclopedia of Genes and Genomes (KEGG) (https://www.kegg.jp) were used to investigate the biological insight and signaling pathways associated with CLDN10 expression in OC. Patients with OC in TCGA database were separated into high and low expression groups according to the median value of CLDN10 expression (5.23) and were retained as the phenotypes. The GSEA software was obtained from the GSEA website (http://software.broadinstitute.org/gsea/index.jsp). A total of 1,000 permutations were selected as the statistical significance to produce valid results. Pathways with a false discovery rate $<0.05$ and $\mathrm{P}<0.05$ were selected as the enriched terms. The molecular signature database from GSEA was utilized as the annotation file.

Statistical analysis. DEGs were identified using the limma package (Bioconductor/R v.3.34) and Wilcoxon test. Htseq-counts (TCGA) and RSEM (GEO, log2 scaled) were used as the sequencing data formats. The nomogram was established using Regression Modeling Strategies (R v3.34). Unpaired Student's t-test and Fisher's exact test were performed in R (v.3.34).

\section{Results}

Identification of DEGs and candidate genes. A total of 269 DEGs were discovered by bioinformatic analysis (differentially expressed in at least 2 datasets), including 32 upregulated and 237 downregulated genes. Venn plots were generated to illustrate the collection of DEGs (Fig. 1A and B). To discover the potential candidate genes to be used for OC prognosis, the Kaplan-Meier method and the log-rank test were performed on the total number of DEGs. A total of 15 genes $(\mathrm{P}<0.05)$ were selected from the total number of DEGs as candidate signatures for further analysis. The median CLDN10 expression level from all OC tissues from the TCGA database was used as the cut-off value to classify the OC tissues into high $(n=133)$ and low $(n=133)$ CLDN10 expression groups. CLDN10 expression exhibited the most significant association with survival time in patients with OC (Fig. 1C). In addition, the expression levels of CLDN10 were upregulated in OC tissues compared with those in normal tissues, as determined by the GEO datasets (Fig. 1D). As shown in Table I, CLDN10 expression was significantly associated with age and overall survival time, while no significant association was identified between CLDN10 expression and tumor grade, tumor pathological stage, primary site, tumor status, vascular invasion index, disease-free survival time and longest diameter. qPCR results 
Table I. Association between CLDN10 expression and clinicopathological characteristics of patients with ovarian cancer.

\begin{tabular}{|c|c|c|c|c|c|}
\hline \multirow[b]{2}{*}{ Clinical factor } & \multicolumn{2}{|c|}{ CLDN10 expression } & \multirow[b]{2}{*}{ Total $(n=266)$} & \multirow[b]{2}{*}{$95 \% \mathrm{CI}$} & \multirow[b]{2}{*}{ P-value } \\
\hline & High $(n=133)$ & Low $(\mathrm{n}=133)$ & & & \\
\hline Mean age \pm SD, years & $57.14 \pm 10.47$ & $60.9 \pm 11.04$ & & $1.1513-6.3675$ & 0.0049 \\
\hline \multicolumn{6}{|l|}{ Tumor pathological stage, $\mathrm{n}$} \\
\hline Stage I-II & 9 & 11 & 20 & $0.4495-3.5211$ & 0.8167 \\
\hline Stage III-IV & 123 & 121 & 244 & & \\
\hline Grade, $n$ & & & $2.8688-3.0024$ & & \\
\hline G1 & 0 & 1 & 1 & 0.8200 & \\
\hline $\mathrm{G} 2$ & 15 & 17 & 32 & & \\
\hline G3 & 113 & 111 & 224 & & \\
\hline G4 & 0 & 1 & 1 & & \\
\hline GB & 1 & 1 & 2 & & \\
\hline GX & 3 & 1 & 4 & & \\
\hline Primary site, $\mathrm{n}$ & & & $0.3413-0.5238$ & & \\
\hline Bilateral & 95 & 85 & 180 & 0.6890 & \\
\hline Left & 16 & 19 & 35 & & \\
\hline Right & 18 & 19 & 37 & & \\
\hline Tumor status & & & $0.7075-1.8518$ & & \\
\hline Tumor-free & 34 & 25 & 59 & 0.6426 & \\
\hline With tumor & 83 & 91 & 174 & & \\
\hline Vascular invasion indicator & & & $0.3081-2.1570$ & & \\
\hline Yes & 15 & 18 & 33 & 0.6596 & \\
\hline No & 21 & 31 & 52 & & \\
\hline Mean disease-free survival time $\pm \mathrm{SD}$, months & $20.74 \pm 20.23$ & $17.139 \pm 12.62$ & & $-7.4010-0.2000$ & 0.0633 \\
\hline Mean overall survival time $\pm \mathrm{SD}$, months & $40.976 \pm 30.86$ & $31.271 \pm 22.46$ & & $-16.5740(-)-2.8350$ & 0.0058 \\
\hline Longest dimension, $\mathrm{cm}$ & 1.353077 & 1.485833 & & $0.0087-0.27426$ & 0.0658 \\
\hline
\end{tabular}

CLDN10, claudin 10 .

demonstrated that CLDN10 expression was upregulated in OC tissues from the hospital cohort compared with that in normal tissues (Fig. 1E). Furthermore, the expression levels of CLDN10 were downregulated in International Federation of Gynecology and Obstetrics (FIGO) III/IV samples compared with those in FIGO I/II samples (Fig. 1F).

CLDN10-associated prognostic nomogram. Patients with OC with low CLDN10 expression had a poor disease prognosis compared with patients with high expression $(\mathrm{P}=0.0018$; Fig. 2A). A prognostic nomogram was established between CLDN10 expression and several significant clinical factors, including age, vascular invasion index and AJCC staging rules (Fig. 2C). Furthermore, calibration curves indicated an excellent performance in prediction (Fig. 2B). In the current nomogram, all variables were in accordance with the clinical data. CLDN10 expression was utilized as a new variable with improved model accuracy. The expression levels of CLDN10 were positively associated with the risk score. The present nomogram further indicated that low CLDN10 expression in patients with OC may result in a low survival rate within 5 years. The C-index of the model was 0.776 .
CLDN10-associated ceRNA network and biological pathways. To fully understand the association between CLDN10 expression and IncRNA-miRNA-circRNA pathways, a ceRNA network associated with CLDN10 expression was established in the present study. A total of 8 miRNAs were identified to be associated with CLDN10, including miR-486-5p, miR-3919, miR-6801-3p, miR-3925-5p, miR-6729-3p, miR-4756-3p, miR-6810-3p and miR-331-3p (Fig. 3). In addition, 13 circRNAs were associated with CLDN10 expression, namely circRNA9920, circRNA84, circRNA657, circRNA15564, circRNA15246, circRNA11697, circRNA9414, circRNA8849, circRNA15229, circRNA2961, circRNA6705, circRNA8065 and circRNA15486. Furthermore, an association between circRNA-miRNA and lncRNA-miRNA was demonstrated. X-inactive specific transcript, KCNQ1 overlapping transcript 1 and nuclear paraspeckle assembly transcript 1 may be considered the key lncRNAs associated with CLDN10 expression. The ceRNA network revealed that miR-486-5p-CLDN10 and miR-3919-CLDN10 may be significantly involved in the signaling pathways of OC.

GSEA. GSEA was applied to elucidate the biological pathways associated with CLDN10 expression in OC. Four 

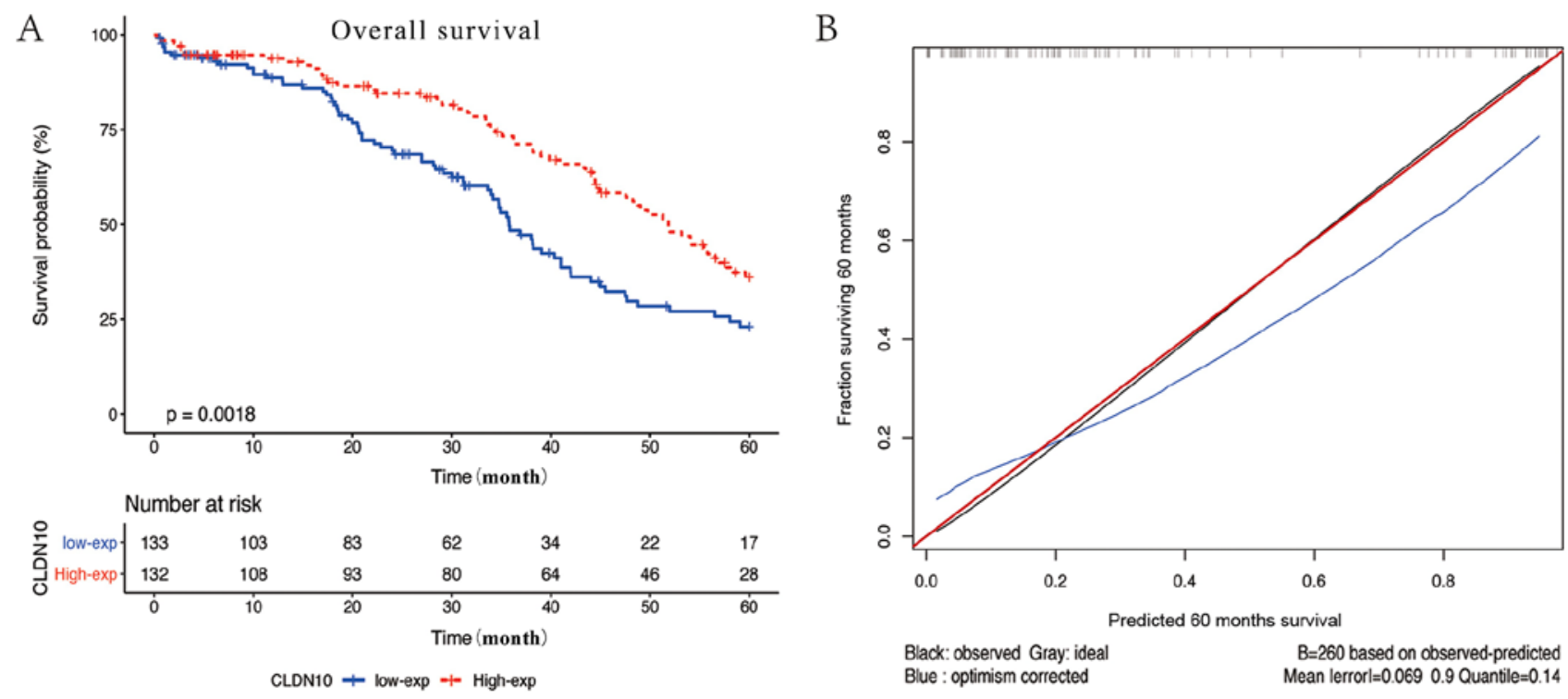
Blue : optimism corrected

C

Points

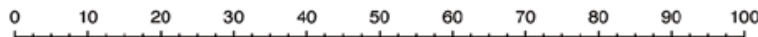

nCLDN10

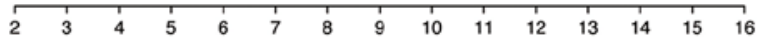

AGE

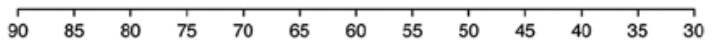

Clinical_Stage

Stage I-II

PRIMARY_SITE

Stage III-IV

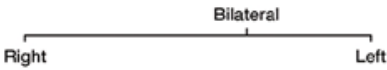

LYMPHOVASCULAR_INVASION_INDICATOR_NO

YES

Total Points

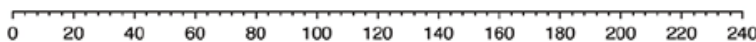

Linear Predictor

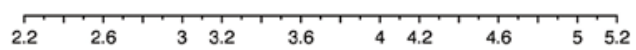

5-year Survival Probability

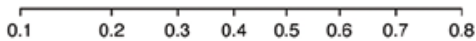

3-year Survival Probability

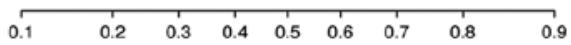

1-year Survival Probability

$0.40 .450 .50 .50 .650 .70 .750 .80 .85 \quad 0.9 \quad 0.95$

Figure 2. Prognostic value of CLDN10 in clinical applications. (A) Overall survival analysis of patients with low and high CLDN10 expression in The Cancer Genome Atlas database. Low expression levels of CLDN10 indicated poor survival. (B) Difference between true values and predicted values. (C) Nomogram for predicting survival probabilities. Each clinical characteristic is shown with its corresponding score. CLDN10 expression was used as a novel variable in the nomogram. nCLDN10 represents the normalized expression levels of CLDN10. The C-index of the nomogram is 0.776. CLDN10, claudin 10.

signaling pathways were associated with CLDN10 expression, including the 'KEGG TGF beta signaling pathway' $(\mathrm{P}=0.00371)$, the 'KEGG basal cell carcinoma' pathway $(\mathrm{P}=0.0000513)$, the 'KEGG WNT signaling pathway' $(\mathrm{P}=0.021978)$ and the 'KEGG melanoma' pathway $(\mathrm{P}=0.022813)$. In addition, 25 signaling pathways were associated with CLDN10 expression as demonstrated by KEGG analysis (Fig. 4E), among which the 'KEGG TGF $\beta$ signaling pathway' and the 'KEGG WNT signaling pathway' were the main targets.

\section{Discussion}

$\mathrm{OC}$ is one of the most fatal gynecological malignancies in the world, possibly due to the late diagnosis and low 5-year survival rate of patients with an advanced stage of the disease $(1,18,19)$. 


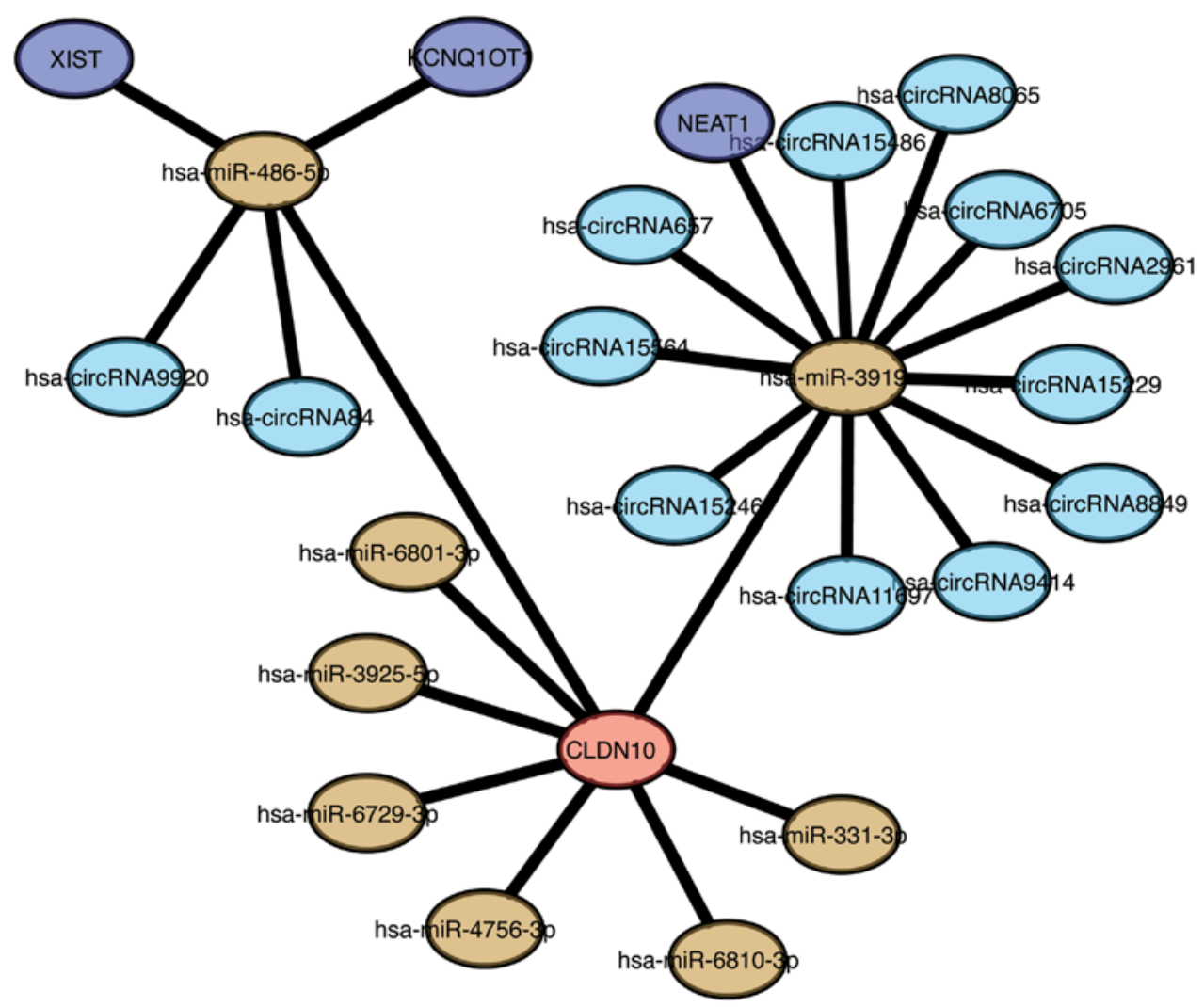

Figure 3. ceRNA network constructed with the Cytoscape software. Red node, CLDN10; yellow nodes, miRs; light blue nodes, circRNAs; blue nodes, long non-coding RNAs. CLDN10, claudin 10; miRs, microRNAs; circRNAs, circular RNAs.

In addition, poor therapeutic responses of patients with $\mathrm{OC}$ have been reported for specific treatments, including chemotherapeutic agents based on platinum salts $(1,18,19)$. Therefore, it is imperative to identify novel prognostic or diagnostic biomarkers with high sensitivity and specificity for early OC screening, monitoring of tumor progression and assessment of the overall therapeutic efficiency or patient risk stratification.

In the present study, GSE66957, GSE6008 and GSE26712 were selected from the GEO database for DEG analysis. A total of 269 DEGs were identified in OC tissues, of which 32 were upregulated and 237 were downregulated compared with the corresponding gene expression levels in normal tissues. The Kaplan-Meier method was used with the log-rank test and nomogram analysis, and the results indicated that low CLDN10 expression in patients with OC may be associated with a low 5-year survival rate. qPCR analysis demonstrated that CLDN10 expression was upregulated in OC tissues compared with that in normal tissues. Notably, the expression levels of CLDN10 were downregulated in FIGO III/IV tumor samples compared with those in FIGO I/II samples. CLDN10 is a member of the claudin family, which influences the progression of several types of cancer, including papillary thyroid cancer, breast cancer, lung adenocarcinoma, ependymomas, esophageal squamous cell carcinoma and hepatocellular carcinoma (20-26). Similarly, lower expression levels of CLDN10 are associated with higher grade ependymomas and invasive lung adenocarcinoma $(23,24)$. However, higher CLDN10 expression is associated with a poor prognosis in papillary thyroid cancer (20). Functional genes serve different roles in different tumors due to the complexity of the tumor, and expression levels of claudins seem to change in a tissue-specific manner (23). Claudins are the major constituents of tight junctions that function as integral membrane proteins; their expression levels are absent or decreased in human neoplastic tissues (27). Tight junctions could be disrupted or 'loosened' in cancer cells in order to induce dissemination and migration during the cancer metastatic process. Therefore, claudins, including CLDN1, CLDN3, CLDN4, CLDN6, CLDN7, CLDN10, CLDN14 and CLDN17, serve a critical role in modulating carcinogenesis and metastasis (28-37). In a previous study, CLDN10 was reported to suppress cell metastasis by phosphorylating the Janus kinase 1/signaling transducer and activator of transcription 1 signaling pathway in osteosarcoma cells (38). However, the cellular mechanisms of CLDN10 in regulating the progression of $\mathrm{OC}$ remain unclear.

In the present study, GSEA was performed to assess CLDN10 expression, and the results indicated that it was significantly associated with the TGF- $\beta$ and WNT signaling pathways. TGF- $\beta$ is a multifunctional cytokine that modulates cell morphogenesis and differentiation, and is the main inducer of EMT during carcinogenesis and fibrosis (39). In addition, WNT signaling is tightly associated with cancer progression and is one of the key cascades responsible for modulating development and stemness of cancer cells. This pathway is divided into the $\beta$-catenin-dependent and -independent signaling pathways (40). The WNT/ $\beta$-catenin signaling pathway is implicated in the carcinogenesis of various types of OC and is involved in EMT (41). Previous studies have demonstrated that CLDN1 serves a major role in regulating EMT and in inducing $\beta$-catenin expression in cancer cells $(42,43)$, indicating that CLDN10 
A

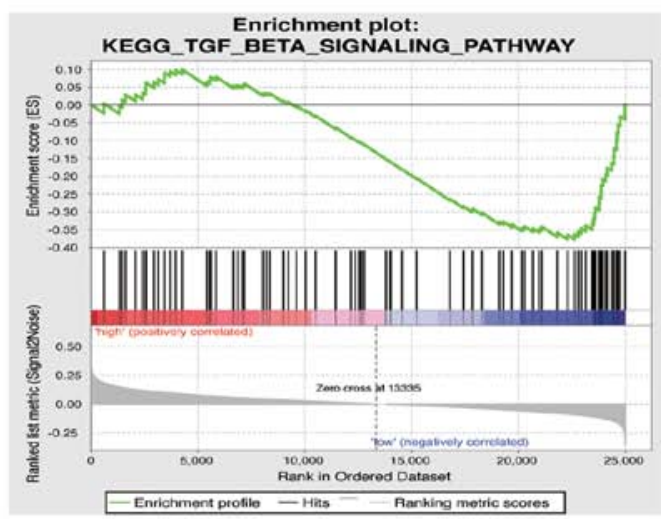

C

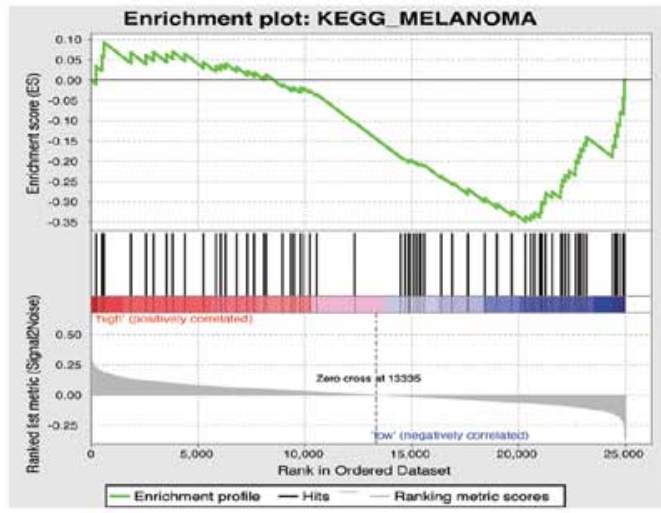

B

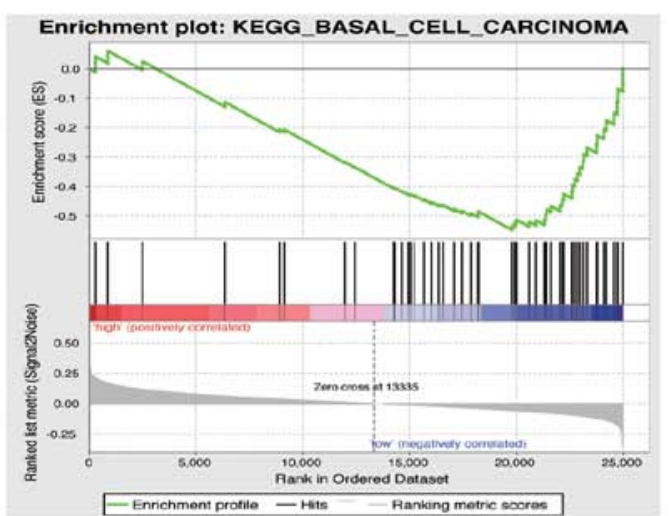

D Enrichment plot:KEGG_WNT_SIGNALING_PATHWAY

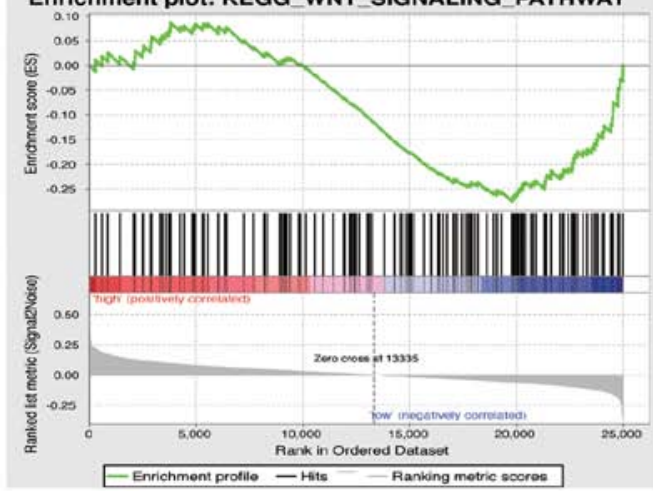

E

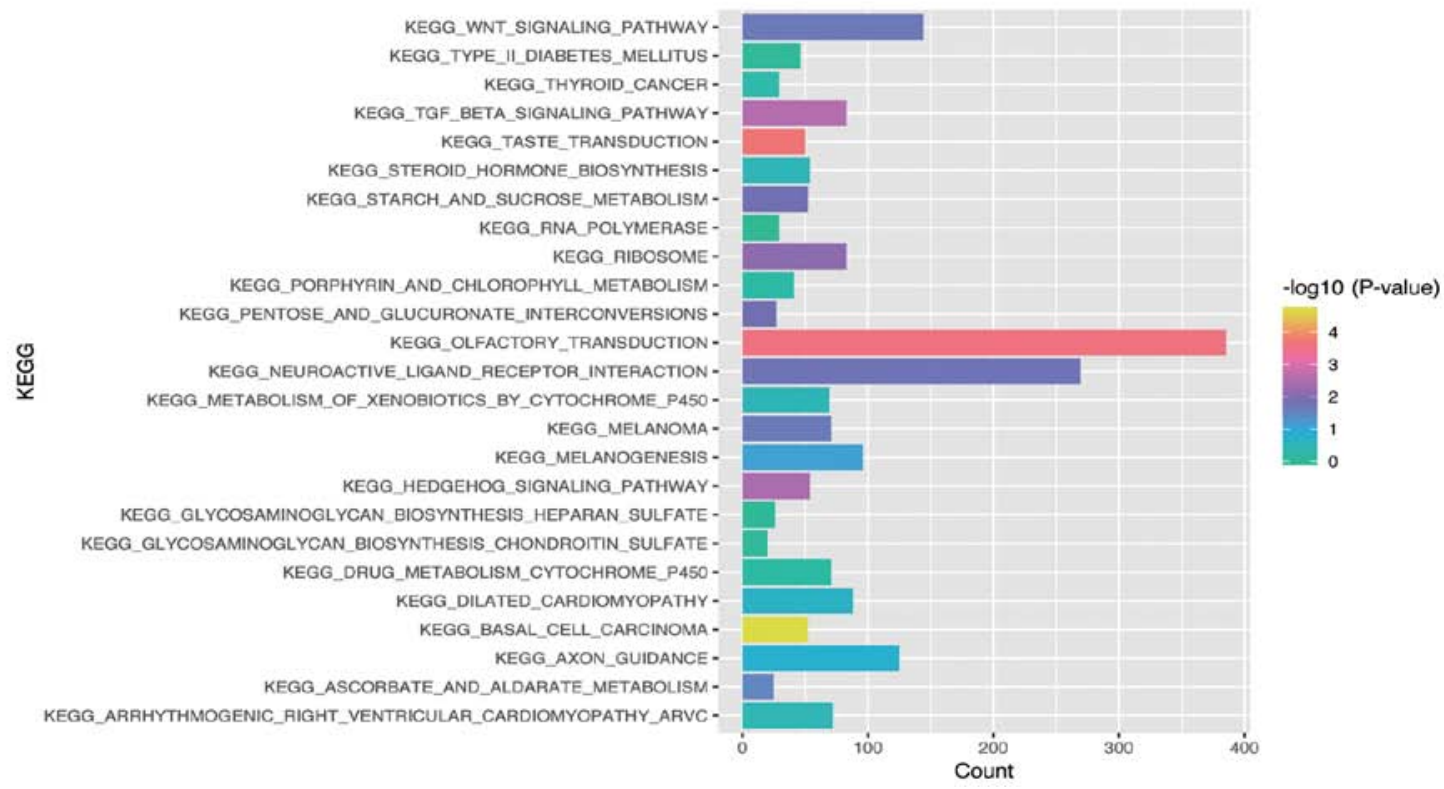

Figure 4. Detection of biological pathways and internal mechanisms. (A) Enrichment of genes in the 'KEGG_TGF_BETA_SIGNALING_PATHWAY' via GSEA. (B) Enrichment of genes in 'KEGG_BASAL_CELL_CARCINOMA' via GSEA. (C) Enrichment of genes in 'KEGG_MELANOMA' via GSEA. (D) Enrichment of genes in the 'KEGG_WNT_SIGNALING_PATHWAY' via GSEA. (E) Enriched pathways identified via GSEA using the Molecular Signatures Database. KEGG, Kyoto Encyclopedia of Genes and Genomes; GSEA, gene set enrichment analysis; TGF, transforming growth factor.

expression in OC may be associated with EMT or $\beta$-catenin. Based on the aforementioned studies, it was hypothesized that CLDN10 may regulate the progression of OC via TGF- $\beta$ - or WNT/ $\beta$-catenin-induced EMT, which should be further investigated in future studies. Furthermore, a CLDN10-associated ceRNA network was constructed in the present study, and the results revealed that miR-486-5p and miR-3919 may be the key regulators of CLDN10 expression in OC. Notably, miR-486 is reported to regulate cancer metastasis in hepatocellular carcinoma by targeting CLDN10, and miR-486-5p could inhibit cell proliferation and apoptosis in renal cell carcinoma via TGF- $\beta$-activated kinase $1(44,45)$. Further investigations on the associations among CLDN10, miR-486-5p, miR-3919, TGF- $\beta$, WNT/ $\beta$-catenin and EMT should be conducted in future 
studies, and could provide valuable insight on the cellular mechanisms underlying OC progression.

In the present study, the results demonstrated that decreased CLDN10 expression could predict lower overall survival rate and time in patients with OC. Furthermore, a predictive nomogram with high accuracy was established for prognosis prediction. GSEA and ceRNA network analyses were performed to elucidate the potential biological processes involving CLDN10, and the results indicated that this gene may regulate the progression of OC via TGF- $\beta$ - or WNT/ $\beta$-catenin-induced EMT. In the present study, the analyzed datasets displayed limited information about the histological subtypes of $\mathrm{OC}$, which was insufficient for statistical analysis. Therefore, the change of expressed genes with differing histological subtype was not assessed, and the association between CLDN10 expression and the histological subtype of OC should be analyzed in future studies. Finally, the association among CLDN10, miR-486-5p, miR-3919, TGF- $\beta$, WNT/ $\beta$-catenin and EMT of OC cells should be further explored in future investigations.

\section{Acknowledgements}

Not applicable.

\section{Funding}

The present study was financially supported by grants from the National Natural Science Foundation of China (grant no. 81702399), the Social Science and Technology Development Key Project of Dongguan City (grant no. 2018507150011651), the Guangdong Province Medical Scientific Research Foundation (grant nos. C2018053 and C2017034) and the National Medical Science and Technology Foundation (grant no. W2016CWGD05).

\section{Availability of data and materials}

The datasets used and/or analyzed during the present study are available from the corresponding author on reasonable request. The expression profiles of the transcriptomes from the following datasets were systematically analyzed to ensure the credibility of the present study: GSE66957 ( $n=69$; Moffitt Cancer Center, Biostatistics and Bioinformatics), GSE6008 ( $\mathrm{n}=103$; University of Michigan) and GSE26712 ( $\mathrm{n}=195$; University of Michigan) (https://www.ncbi.nlm.nih. gov/geo/query/acc.cgi). The clinicopathological characteristics of 266 patients with OC were collected from TCGA database via the cBioPortal (http://www.cbioportal.org).

\section{Authors' contributions}

SH and ZL conceived and designed the experiments. SH, ZL and WX performed the experiments and data analysis, and drafted the manuscript. $\mathrm{LH}, \mathrm{NC}, \mathrm{ZH}, \mathrm{BL}$ and $\mathrm{CW}$ participated in data collection and analysis. All authors read and approved the final manuscript.

\section{Ethics approval and consent to participate}

The present study was approved by the Ethics Committee of Dongguan Affiliated Hospital, Southern Medical University
(Dongguan, China), and informed consent was obtained from all patients in the hospital cohort. The experiments performed in the present study involved human specimens and were in accordance with the Declaration of Helsinki.

\section{Patient consent for publication}

Not applicable.

\section{Competing interests}

The authors declare that they have no competing interests.

\section{References}

1. Siegel RL, Miller KD and Jemal A: Cancer statistics, 2019. CA Cancer J Clin 69: 7-34, 2019.

2. Testa U, Petrucci E, Pasquini L, Castelli G and Pelosi E: Ovarian cancers: Genetic abnormalities, tumor heterogeneity and progression, clonal evolution and cancer stem cells. Medicines (Basel) 5: pii: E16, 2018.

3. Meinhold-Heerlein I and Hauptmann S: The heterogeneity of ovarian cancer. Arch Gynecol Obstet 289: 237-239, 2014.

4. Kim S, Han Y, Kim SI, Kim HS, Kim SJ and Song YS: Tumor evolution and chemoresistance in ovarian cancer. NPJ Precis Oncol 2: 20, 2018.

5. Miller KD, Siegel RL, Lin CC, Mariotto AB, Kramer JL, Rowland JH, Stein KD, Alteri R and Jemal A: Cancer treatment and survivorship statistics, 2016. CA Cancer J Clin 66: 271-289, 2016.

6. Heintz AP, Odicino F, Maisonneuve P, Quinn MA, Benedet JL, Creasman WT, Ngan HY, Pecorelli S and Beller U: Carcinoma of the ovary. FIGO 26th annual report on the results of treatment in gynecological cancer. Int J Gynaecol Obstet 95 (Suppl 1): S161-S192, 2006.

7. Berek JS, Kehoe ST, Kumar L and Friedlander M: Cancer of the ovary, fallopian tube, and peritoneum. Int J Gynaecol Obstet 143 (Suppl 2): S59-S78, 2018.

8. Altman DG and Riley RD: Primer: An evidence-based approach to prognostic markers. Nat Clin Pract Oncol 2: 466-472, 2005.

9. Gan Y, Li Y, Li T, Shu G and Yin G: CCNA2 acts as a novel biomarker in regulating the growth and apoptosis of colorectal cancer. Cancer Manag Res 10: 5113-5124, 2018.

10. Kulasingam V and Diamandis EP: Strategies for discovering novel cancer biomarkers through utilization of emerging technologies. Nat Clin Pract Oncol 5: 588-599, 2008.

11. Liu J, Meng H, Li S, Shen Y, Wang H, Shan W, Qiu J, Zhang J and Cheng W: Identification of potential biomarkers in association with progression and prognosis in epithelial ovarian cancer by integrated bioinformatics analysis. Front Genet 10: 1031, 2019.

12. Wu R, Zhai Y, Kuick R, Karnezis AN, Garcia P, Naseem A, $\mathrm{Hu}$ TC, Fearon ER and Cho KR: Impact of oviductal versus ovarian epithelial cell of origin on ovarian endometrioid carcinoma phenotype in the mouse. J Pathol 240: 341-351, 2016.

13. Yang X, Zhu S, Li L, Zhang L, Xian S, Wang Y and Cheng Y: Identification of differentially expressed genes and signaling pathways in ovarian cancer by integrated bioinformatics analysis. Onco Targets Ther 11: 1457-1474, 2018.

14. Liu Y, Kuick R, Hanash S and Richardson B: DNA methylation inhibition increases T cell KIR expression through effects on both promoter methylation and transcription factors. Clin Immunol 130: 213-224, 2009.

15. Gomes Ferreira M, Sancho de Salas M, González Sarmiento R and Doyague Sánchez MJ: Changes in the management and prognosis of ovarian cancer due to the new FIGO and WHO classifications: A case series observational descriptive study. Seven years of follow-up. Int J Gynecol Cancer 28: 1461-1470, 2018.

16. Zeppernick F and Meinhold-Heerlein I: The new FIGO staging system for ovarian, fallopian tube, and primary peritoneal cancer. Arch Gynecol Obstet 290: 839-842, 2014.

17. Livak KJ and Schmittgen TD: Analysis of relative gene expression data using real-time quantitative PCR and the 2(-Delta Delta C(T)) method. Methods 25: 402-408, 2001. 
18. Lengyel E: Ovarian cancer development and metastasis. Am J Pathol 177: 1053-1064, 2010.

19. Torre LA, Trabert B, DeSantis CE, Miller KD, Samimi G, Runowicz CD, Gaudet MM, Jemal A and Siegel RL: Ovarian cancer statistics, 2018. CA Cancer J Clin 68: 284-296, 2018.

20. Zhou Y, Xiang J, Bhandari A, Guan Y, Xia E, Zhou X, Wang Y and Wang O: CLDN10 is associated with papillary thyroid cancer progression. J Cancer 9: 4712-4717, 2018.

21. Liao J, Li J, Cheng H, Chen Y and Mo Z: CLDN10 single nucleotide polymorphism rs1325774 alters the risk of breast cancer in south chinese women. Medicine (Baltimore) 97: e13187, 2018.

22. Huang GW, Ding X, Chen SL and Zeng L: Expression of claudin 10 protein in hepatocellular carcinoma: Impact on survival. J Cancer Res Clin Oncol 137: 1213-1218, 2011.

23. Nordfors K, Haapasalo J, Sallinen PK, Haapasalo H and Soini Y: Expression of claudins relates to tumour aggressivity, location and recurrence in ependymomas. Histol Histopathol 28: 1137-1146, 2013.

24. Zhang Z, Wang A, Sun B, Zhan Z, Chen K and Wang C: Expression of CLDN1 and CLDN10 in lung adenocarcinoma in situ and invasive lepidic predominant adenocarcinoma. J Cardiothorac Surg 8: 95, 2013.

25. Chattopadhyay I, Singh A, Phukan R, Purkayastha J, Kataki A, Mahanta J, Saxena S and Kapur S: Genome-wide analysis of chromosomal alterations in patients with esophageal squamous cell carcinoma exposed to tobacco and betel quid from high-risk area in India. Mutat Res 696: 130-138, 2010.

26. Barros-Filho MC, Marchi FA, Pinto CA, Rogatto SR and Kowalski LP: High diagnostic accuracy based on CLDN10, HMGA2, and LAMB3 transcripts in papillary thyroid carcinoma. J Clin Endocrinol Metab 100: E890-E899, 2015.

27. Osanai M, Takasawa A, Murata M and Sawada N: Claudins in cancer: Bench to bedside. Pflugers Arch 469: 55-67, 2017.

28. Nichols LS, Ashfaq R and Iacobuzio-Donahue CA: Claudin 4 protein expression in primary and metastatic pancreatic cancer: Support for use as a therapeutic target. Am J Clin Pathol 121: 226-230, 2004

29. Chao YC, Pan SH, Yang SC, Yu SL, Che TF, Lin CW, Tsai MS, Chang GC, Wu CH, Wu YY, et al: Claudin-1 is a metastasis suppressor and correlates with clinical outcome in lung adenocarcinoma. Am J Respir Crit Care Med 179: 123-133, 2009.

30. Resnick MB, Konkin T, Routhier J, Sabo E and Pricolo VE: Claudin-1 is a strong prognostic indicator in stage II colonic cancer: A tissue microarray study. Mod Pathol 18: 511-518, 2005.

31. Tokés AM, Kulka J, Paku S, Szik A, Páska C, Novák PK, Szilák L, Kiss A, Bögi K and Schaff Z: Claudin-1, -3 and -4 proteins and mRNA expression in benign and malignant breast lesions: A research study. Breast Cancer Res 7: R296-R305, 2005.

32. Guo Y, Lin D, Zhang M, Zhang X, Li Y, Yang R, Lu Y, Jin X, Yang $M$ and Wang M, et al: CLDN6-induced apoptosis via regulating ASK1-p38/JNK signaling in breast cancer MCF-7 cells. Int J Oncol 48: 2435-2444, 2016.
33. Zhou S, Piao X, Wang C, Wang R and Song Z: Identification of claudin-1, $-3,-7$ and -8 as prognostic markers in human laryngeal carcinoma. Mol Med Rep 20: 393-400, 2019.

34. Kominsky SL, Argani P, Korz D, Evron E, Raman V, Garrett E, Rein A, Sauter G, Kallioniemi OP and Sukumar S: Loss of the tight junction protein claudin-7 correlates with histological grade in both ductal carcinoma in situ and invasive ductal carcinoma of the breast. Oncogene 22: 2021-2033, 2003.

35. Honda H, Pazin MJ, D'Souza T, Ji H and Morin PJ: Regulation of the CLDN3 gene in ovarian cancer cells. Cancer Biol Ther 6: 1733-1742, 2007.

36. Gao M,Li W, Wang Hand Wang G: The distinct expression patterns of claudin-10, -14, -17 and E-cadherin between adjacent non-neoplastic tissues and gastric cancer tissues. Diagn Pathol 8: 205, 2013.

37. Tabariès $\mathrm{S}$ and Siegel PM: The role of claudins in cancer metastasis. Oncogene 36: 1176-1190, 2017.

38. Zhang X, Wang X, Wang A, Li Q, Zhou M and Li T: CLDN10 promotes a malignant phenotype of osteosarcoma cells via JAK1/Stat1 signaling. J Cell Commun Signal 13: 395-405, 2019.

39. Willis BC and Borok Z: TGF-beta-induced EMT: Mechanisms and implications for fibrotic lung disease. Am J Physiol Lung Cell Mol Physiol 293: L525-L534, 2007.

40. Zhan T, Rindtorff N and Boutros M: Wnt signaling in cancer. Oncogene 36: 1461-1473, 2017.

41. Arend RC, Londoño-Joshi AI, Straughn JJ Jr and Buchsbaum DJ: The Wnt/ $\beta$-catenin pathway in ovarian cancer: A review. Gynecol Oncol 131: 772-779, 2013.

42. Suh Y, Yoon CH, Kim RK, Lim EJ, Oh YS, Hwang SG, An S, Yoon G, Gye MC, Yi JM, et al: Claudin-1 induces epithelial-mesenchymal transition through activation of the c-Abl-ERK signaling pathway in human liver cells. Oncogene 32: 4873-4882, 2013.

43. Wu X, Xiao J, Zhao C, Zhao C, Han Z, Wang F, Yang Y, Jiang Y and Fang F: Claudin1 promotes the proliferation, invasion and migration of nasopharyngeal carcinoma cells by upregulating the expression and nuclear entry of $\beta$-catenin. Exp Ther Med 16: 3445-3451, 2018.

44. Sun H, Cui C, Xiao F, Wang H, Xu J, Shi X, Yang Y, Zhang Q, Zheng $\mathrm{X}$, Yang $\mathrm{X}$, et al: miR-486 regulates metastasis and chemosensitivity in hepatocellular carcinoma by targeting CLDN10 and CITRON. Hepatol Res 45: 1312-1322, 2015.

45. He Y, Liu J, Wang Y, Zhu X, Fan Z, Li C, Yin H and Liu Y: Role of miR-486-5p in regulating renal cell carcinoma cell proliferation and apoptosis via TGF- $\beta$-activated kinase 1 . J Cell Biochem 120: 2954-2963, 2019.

This work is licensed under a Creative Commons Attribution-NonCommercial-NoDerivatives 4.0 International (CC BY-NC-ND 4.0) License. 